\title{
Verhalten von Nanosilber in Kläranlagen und dessen Einfluss auf die Nitrifikationsleistung in Belebtschlamm
}

\author{
Michael Burkhardt $\cdot$ Steffen Zuleeg $\cdot$ Ralf Kägi $\cdot$ Brian Sinnet · Jakob Eugster $\cdot$ Markus Boller $\cdot$ Hansruedi Siegrist
}

Erhalten: 20. April 2010/Akzeptiert: 25. August 2010/Online veröffentlicht: 24. September 2010

(C) Springer-Verlag 2010

Zusammenfassung Hintergrund, Ziel und Zweck Die Anwendung von Nanosilber nimmt zwar stetig zu, doch die Kenntnisse zum Vorkommen nanoskaligen Silbers in Abwässern und zum Verhalten in Kläranlagen sind gering. Untersuchungen unter realen Betriebsbedingungen fehlen sogar vollständig. In der vorliegenden Studie wurde Nanosilber mit folgenden Zielen untersucht: 1) Hemmung der Nitrifikationsleistung im Belebtschlamm einer Kläranlage, 2) Erstellung einer Massenbilanz für Nanosilber in einer Pilotkläranlage und 3) Verifizierung der Bilanz durch eine Untersuchung in einer realen kommunalen Kläranlage, in die durch einen Indirekteinleiter partikuläres Silber eingetragen wird.

Methoden Der Einfluss von vier marktüblichen Nanosilberadditiven auf die Ammoniumoxidation im Belebtschlamm wurde in Batchreaktoren bei zwei Konzentrationen (1, $100 \mathrm{mg} / \mathrm{L} \mathrm{Ag)} \mathrm{und} \mathrm{zwei} \mathrm{Expositionszeiten} \mathrm{(2} \mathrm{h,}$ 6 Tage) untersucht. In einer Pilotkläranlage in der Größe von 70 Einwohnerwerten wurde partikuläres Silberchlorid und metallisches Nanosilber jeweils für die Dauer zweier Schlammalter (25 Tage) dosiert und weitere zwei Schlammalter bilanziert. Als Grundlage wurden die Abwassermen-

M. Burkhardt $\cdot$ S. Zuleeg $\cdot$ R. Kägi $\cdot$ B. Sinnet $\cdot$ J. Eugster $\cdot$ M. Boller $\cdot$ H. Siegrist

Eawag: Das Wasserforschungs-Institut des ETH-Bereichs,

Überlandstraße 133, 8600 Dübendorf, Schweiz

M. Burkhardt $(\bowtie)$

HSR Hochschule für Technik,

Institut für Umwelt- und Verfahrenstechnik (UMTEC),

Oberseestraße 10, 8640 Rapperswil, Schweiz

E-Mail: michael.burkhardt@hsr.ch

S. Zuleeg

KUSTER + HAGER Ingenieurbüro AG,

Oberstrasse 222, 9014 St. Gallen, Schweiz gen und die Silberkonzentrationen erfasst. Zudem wurde die Anlagerung von Nanosilber an Feststoffen durch elektronenmikroskopische Untersuchungen erfasst. Auf einer Kläranlage (60000 Einwohnerwerte) wurden der Zu- und Ablauf sowie der Faulschlamm im Rahmen von vier Messkampagnen beprobt und wiederum auf Silber chemisch und Nanosilber mikroskopisch analysiert.

Ergebnisse Die Zugabe von $1 \mathrm{mg} / \mathrm{L} \mathrm{Ag}$ in Form von Silbernitrat, metallischem Nanosilber, partikulärem Silberchlorid und Mikrokomposit-Silber bleibt ohne Wirkung auf die Nitrifikationsleistung. Dagegen resultieren $100 \mathrm{mg} / \mathrm{L}$ Ag für alle Silberformen in einer Hemmung, welche zwischen 100\% für Silbernitrat und 20-30\% für kolloidales Nanosilber liegt. In der Massenbilanzstudie wird deutlich, dass nach Zugabe von partikulärem Silberchlorid 96\% des Silbers mit dem Schlamm und $4 \%$ über das Nachklärbecken - also das gereinigte Abwasser - ausgetragen werden. Die Massenbilanz für metallisches Nanosilber fällt ähnlich aus. Diese Bilanzen aus der Pilotkläranlage decken sich mit derjenigen von einer realen Kläranlage. Der Silberaustrag korreliert mit dem Feststoffaustrag im Schlammabzug und Nachklärbeckenablauf. Die Jahresfracht zum Vorfluter dürfte bei 4-40 mg/a Ag pro Einwohnerwert liegen. Mit Elektronenmikroskopie lässt sich zeigen, dass das Nanosilber in und an die Belebtschlammflocken gebunden vorliegt.

Diskussion Chlorid ist im Abwasser in größeren Mengen verfügbar, sodass Silberionen praktisch vollständig zu Silberchlorid ausfallen. Silberionen binden auch an organischen Komplexbildnern und reduzierten Schwefelgruppen. Deshalb ist eine hemmende Wirkung auf Nitrifikanten selbst für Silbernitrat bei $1 \mathrm{mg} / \mathrm{L} \mathrm{Ag} \mathrm{(250} \mathrm{mg} \mathrm{Ag/kg} \mathrm{TS)} \mathrm{nicht} \mathrm{zu}$ beobachten. Bei der sehr hohen Dosierung von $100 \mathrm{mg} / \mathrm{LAg}$ stellt sich nicht genügend schnell ein Lösungsgleichgewicht zwischen freien Silberionen und Bindungsstellen ein. Die Massenbilanz über die Kläranlage unterstreicht, dass $\mathrm{Na}$ - 
nosilber sehr gut an Partikeln bindet und effizient aus dem Abwasser eliminiert wird. Verglichen mit problematischen organischen und anorganischen Mikroverunreinigungen ist die Eliminationsrate mit über $95 \%$ hoch. Mit der Reduktion der ungelösten Stoffe (GUS) lässt sich auch die Silberfracht im Ablauf vermindern.

Schlussfolgerungen Nanosilber liegt in der Regel gebunden vor, wird dadurch effizient eliminiert und kann allenfalls mit einer Abwassernachbehandlung in Form einer Raum- oder Flächenfiltration weiter reduziert werden. Der Schlamm stellt die wichtigste Senke für Nanosilber dar. Silberpartikel $>100 \mathrm{~nm}$ Größe binden tendenziell weniger gut als Nanosilber im engeren Sinne. Alle analysierten Silberpartikel im Zulauf, Ablauf und Schlamm deuten darauf hin, dass das Silber vor allem sulfidisch (als $\mathrm{Ag}_{2} \mathrm{~S}$ ) die Kläranlage verlässt. Aufgrund der vernachlässigbaren Wasserlöslichkeit dieser Silberform werden keine Silberionen freigesetzt.

Empfehlungen und Ausblick Die Forschung ist auf Prozessmechanismen und die Oberflächenmodifikation von Nanosilber zu konzentrieren, die die Speziierung und das Bindungsvermögen unter Umweltbedingungen beeinflussen. Verglichen mit anderen organischen Spurenstoffen zeigt sich kein dringender Handlungsbedarf bezüglich Nanosilber im Bereich von Kläranlagen. Maßnahmen zur Emissionsminderung sollten dennoch dem Vorsorgeprinzip folgend entwickelt werden.

Schlüsselwörter Abwasserreinigung · Elimination · Kläranlage $\cdot$ Massenbilanz $\cdot$ Nanosilber . Nitrifikationsleistung $\cdot$ Silberionen $\cdot$ Trockensubstanz

\section{Fate of nanosilver in wastewater treatment plants and their impact on nitrification activity in sewage sludge}

\begin{abstract}
Background, aim and scope The application of nanosilver is increasing. Knowledge on the fate and behavior of nanosilver in wastewater and wastewater treatment plants is scarce. Studies under real world conditions are completely lacking. We studied (1) the impact of nanosilver on the nitrification of sewage sludge, (2) quantified the mass flow of nanosilver in a pilot-plant, and (3) verified the mass balance in a full-scale municipal wastewater treatment plant where nanosilver is introduced to the municipal plant by an indirect discharger.

Materials and Methods The addition of four different nanosilver additives on ammonia oxidation in activated sludge has been studied in batch-reactors at two concentrations (1, $100 \mathrm{mg} / \mathrm{L} \mathrm{Ag}$ ) with two exposure times ( $2 \mathrm{~h}, 6$ days). The pilot-plant treating 70 population equivalents of domestic wastewater is operated with a 12 day sludge age. Nanosilver was applied to the activated sludge tank within two sludge
\end{abstract}

ages. The silver concentrations were measured in sludge and effluent samples during dosing and the following two sludge ages. The adsorption and speciation of silver particles has been analyzed using scanning electron microscopy (SEM) coupled with energy dispersive X-ray spectroscopy (EDX). Influent, effluent and sludge were sampled on a fullscale plant (60000 equivalent inhabitants) and analyzed for silver.

Results Silver nitrate, metallic nanosilver, nano-scaled silver chloride and microcomposite silver did not show any effect on ammonia oxidation after the addition of $1 \mathrm{mg} / \mathrm{L} \mathrm{Ag}$ to the activated sludge (corresponding to $250 \mathrm{mg} \mathrm{Ag}$ per $\mathrm{kg}$ solids). In contrast, $100 \mathrm{mg} / \mathrm{L} \mathrm{Ag}$ inhibited the nitrification process by $100 \%$ after the addition of silver nitrate and 20 $30 \%$ after addition of colloidal polymer-coated nanosilver. A complete mass balance of the pilot-plant, a steady-state system with known fluxes, demonstrates significant enrichment of silver in the sewage sludge $(96 \%)$ after the addition of silver chloride to the plant and small losses of silver into the secondary effluent $(4 \%)$. The mass fluxes are similar to metallic colloidal nanosilver investigated under the same conditions. The mass balance has been consistently confirmed by the full-scale study in a municipal wastewater treatment plant. The silver fluxes correspond to the fluxes of the suspended solids in sludge and effluent. Overall, it is estimated that from public wastewater treatment plants about 4-40 mg/a Ag per inhabitant equivalent are discharged annually to the receiving water. The analysis by SEM-EDX demonstrates adsorption and incorporation of nanosilver on biological flocs. This method yields first insight into complex building and transformation of silver associated with sulfide after adding metallic nanosilver and silver chloride to wastewater.

Discussion Silver ions released from nanosilver react immediately with large amounts of chloride present in wastewater to form silver chloride. Silver ions may react with organic ligands or sulfide groups additionally. Consequently, even silver nitrate added with $1 \mathrm{mg} / \mathrm{L} \mathrm{Ag}(250 \mathrm{mg} \mathrm{Ag} / \mathrm{kg}$ TS) to activated sludge did not inhibit nitrification activity. Very high amounts of nanosilver, i. e. $100 \mathrm{mg} / \mathrm{L} \mathrm{Ag}$, overburdened the system and equilibrium condition between silver ion release and ligands was not reached. The mass balance reflects the excellent attachment of nanosilver to activated sludge and biological flocs. Therefore, the main elimination process of nanosilver is attachment to the activated sludge. The elimination of nanosilver is high compared to organic and inorganic micro pollutants omnipresent in wastewater. Any further reduction of suspended solids in the effluent water will reduce the silver load.

Conclusions Generally, in wastewater nanosilver occurs bonded to activated sludge flocs and therefore the elimination of nanosilver is efficient under operation conditions typical for wastewater treatment plants. The major fraction 
of nanosilver is removed from the system by the excess sludge withdrawal. Nonetheless, the efficiency may be further improved by a tertiary filtration step. All analyses of influent, effluent and sludge confirmed that silver exists as silver sulfide. Due to a negligible water solubility of this silver species, silver ions are not subsequently released.

Recommendations and perspectives Nanosilver research should be driven to the identification of transformation processes in real environmental matrices and the influence of coatings on the adsorption behavior. There is no need for action to take special measures for nanosilver removal in the area of municipal wastewater treatment plants compared to ubiquitous hazardous organic pollutants may present in wastewater. Nevertheless, source control measures following the precautionary principle should be performed.

Keywords Dry matter - Elimination · Mass balance · Nanosilver $\cdot$ Nitrification activity $\cdot$ Silver ion $\cdot$ Wastewater treatment $\cdot$ Wastewater treatment plant

\section{Einleitung}

Die Anwendung von Silber als antimikrobiell wirksames Schutzmittel in Kosmetikprodukten, Kunststoffverpackungen, Desinfektionsmitteln, Farben oder Textilien nimmt zu (Hund-Rinke et al. 2008; Luoma 2008). In den meisten Produkten wird Silber $(\mathrm{Ag})$ in partikulärer Form eingesetzt. Die antimikrobielle Wirkung beruht auf den Silberionen, die in Kontakt mit Feuchtigkeit bzw. Wasser freigesetzt werden (Ratte 1999; Morones et al. 2009). Biozide Silberanwendungen umfassen rund $1 \%(=250 \mathrm{t} / \mathrm{a} \mathrm{Ag})$ des weltweiten Silberverbrauchs (Silverinstitute 2010). Der Verbrauch von metallischem Nanosilber wird auf $0,3 \%(=75 \mathrm{t} / \mathrm{a} \mathrm{Ag})$ geschätzt. Auf die Einwohnerzahl der Schweiz bezogen werden über alle Anwendungsbereiche betrachtet bis zu 2t/a Nanosilber eingesetzt (Müller und Nowack 2008). Jede Silberapplikation mit dem Ziel, ein Produkt antimikrobiell auszurüsten, ist gemäß Biozidprodukterichtlinie zulassungspflichtig (Europäische Union 1998). Dies gilt auch für nanopartikuläres Silber. Darüber hinaus unterliegt die Anwendung von Nanopartikeln keiner gesonderten Zulassung. Die in Anlehnung an die Biozidprodukterichtlinie und für eine Umweltrisikobeurteilung erforderlichen experimentell erarbeiteten Kenntnisse, die explizit auf Nanosilber abzielen, sind gegenwärtig nur lückenhaft verfügbar. Allerdings dürften sich nicht alle Kenntnislücken mit der gleichen Relevanz verbinden.

Von besonders hohem Interesse für Hersteller und Behörden sind vor allem Anwendungen und Expositionspfade in Verbindung mit der aquatischen Umwelt. Für die Beurteilung von Nanosilber im Abwasser sind prioritär Anwendungen im Blickpunkt, bei denen große Einsatzmengen zu erwarten sind, die regelmäßig in Kontakt mit Wasser kommen und deren Abwasserentsorgung an kommunale Kläranlagen angeschlossen ist (Blaser et al. 2008). Dazu gehört der Einsatz in Kosmetikprodukten, Sanitärreinigern und Textilien. Mengenmäßig unbedeutend sind dagegen Außenfarben (Kägi et al. 2010).

Zur antimikrobiellen Veredelung von Textilien werden vor allem nanoskalige Silberformen gewählt, in der Regel Silbersalze (Silberchlorid), Mikrokomposit-Silber, Silberionentauscher in Nanopartikeln und metallisches kolloidales Nanosilber $\left(\mathrm{Ag}^{0}\right)$ (Lee et al. 2003; Gorensek und Recelj 2007; Som et al. 2009). Diesem Anwendungssegment lassen sich weltweit $10 \%(=7,5 \mathrm{t} / \mathrm{a} \mathrm{Ag})$ des Nanosilberverbrauchs zuordnen (Blaser et al. 2008). In der Schweiz wird der Verbrauch für Textilien auf rund $50 \mathrm{~kg}$ Nanosilber pro Jahr geschätzt. Nanosilber, hauptsächlich in Medizinalprodukten zur Infektionsbekämpfung verwendet, wird in Textilien mittels Masterbatches direkt in die Faser eingesponnen oder mittels Textilveredelung in einem Polymersystem auf den Fasern verankert (Som et al. 2009). Genauso werden die in $90 \%$ aller Textilanwendungen eingesetzten Silbersalze und Silberionentauscher appliziert. Eine Veredelung mit Nanosilber wird nur in $10 \%$ der Anwendungen realisiert, denn die Integration von Nanosilber braucht zwar am wenigsten Wirkstoff, kostet aber rund dreimal mehr. Untersuchungen zeigen, dass in Abhängigkeit von Silberform, Oberflächenmodifikation und Materialbeschaffenheit der Textilfasern die applizierten Silberpartikel und gelöstes Silber zu 0-35\% ausgewaschen werden (Kumar et al. 2005; Benn und Westerhoff 2008; Geranio et al. 2009). Diese Silberfracht gelangt über die Kanalisation zur kommunalen Kläranlage. Nachgewiesen wurde auch, dass aus Außenfarbe Nanosilber freigesetzt wird (Burkhardt et al. 2009). Andere Nanosilberanwendungen, die in Kontakt mit Wasser kommen, lassen ebenfalls eine gewisse Auswaschung erwarten.

Experimentell abgesicherte Massenbilanzen für Nanosilber in Kläranlagen fehlen aber. Dies ist darauf zurückzuführen, dass einerseits elektronenmikroskopische Methoden zur Identifizierung von Nanosilber nicht routinemäßig eingesetzt werden, andererseits die Überwachung des Abwassers auf Silber unzureichend ist. Mit dem Rückgang der Einleitungen aus der Fotoindustrie in den 1990er-Jahren wurde die Silbererfassung reduziert.

Zur groben Orientierung lassen sich die Silberkonzentrationen und -frachten im Zulauf von Kläranlagen in Anlehnung an Messungen des Gesamtsilbers ableiten. Beispielsweise resultiert auf Basis einer von Shafer et al. (1998) berichteten mittleren Silberkonzentration von $4 \mu \mathrm{g} / \mathrm{L} \mathrm{Ag}$ im Zulauf von Kläranlagen und einer durchschnittlichen Abwassermenge von jährlich $80 \mathrm{~m}^{3} / \mathrm{a}$ pro Person in der Schweiz für 10 Mio. Einwohnerwerte (gewerbliche Indirekteinleiter eingeschlossen) eine Zulauffracht von $3 \mathrm{t} / \mathrm{a} \mathrm{Ag}$. Eine weitere Worst-Case-Bilanz für den Zulauf basiert auf 
einer mittleren Zulauffracht von 2,5 mg/Tag Ag pro Einwohner (Firk 1986). Diese Abschätzung führt, bei wiederum 10 Mio. Einwohnerwerten, zu einer Jahresfracht von rund 9 t/a Gesamtsilber. Bezogen auf die Schweizer Verbrauchsmenge von maximal $2 \mathrm{t} / \mathrm{a}$ Nanosilber und die in der Regel geringen Freisetzungsraten dürfte damit klar sein, dass nur ein geringer Massenanteil auf das ursprünglich eingesetzte Nanosilber zurückzuführen ist.

Wie gut Nanosilber in Kläranlagen aus dem Abwasser entfernt wird, lässt sich experimentell abgestützt nicht beurteilen, doch deutet die Gesamtsilberelimination von 94-99,9\% auf eine gute Reinigungsleistung hin (Shafer et al. 1998; Howe und Dobson 2002; Benn und Westerhoff 2008). Die Silberkonzentrationen im gereinigten Abwasser von Kläranlagen $(0,001-0,1 \mu \mathrm{g} / \mathrm{L} \mathrm{Ag})$ stehen im Einklang mit der hohen Eliminationsrate und erklären zudem die vergleichsweise geringe Sedimentbelastung in Fließgewässern (Wen et al. 1997; Warila et al. 2001; Benn und Westerhoff 2008; Blaser et al. 2008). Demnach reichert sich Silber bevorzugt im Schlamm mit 1-10 mg Ag/ $\mathrm{kg}$ an; nur industrielle Einleitungen verbinden sich mit höheren Silbergehalten bis $100 \mathrm{mg} \mathrm{Ag} / \mathrm{kg}$ TS (Firk 1986; Bachmann et al. 1999; Howe und Dobson 2002; Blaser et al. 2008). Aktuelle Untersuchungen aus dem Kanton Zürich bestätigen den durchschnittlichen Belastungsbereich (AWEL 2010).

Im realen Belebtschlamm konnte bis $50 \mathrm{mg} \mathrm{Ag} / \mathrm{kg}$ TS Silberbelastung kein messbarer Einfluss auf die Nitrifikationsleistung gezeigt werden (Firk 1986; Howe und Dobson 2002). Choi et al. (2008) setzten dagegen konditionierten Belebtschlamm ein, der zuvor von potenziellen Liganden wie Clorid- und Sulfationen gereinigt wurde $(<1 \mathrm{mg} / \mathrm{L})$, und wiesen daher nach Zugabe von $1 \mathrm{mg} / \mathrm{L}$ Ag (als kolloidales Nanosilber und partikuläres Silberchlorid) eine signifikante Hemmung nach.

Abzuklären gilt es folglich, wie sich Nanosilber in Kläranlagen verhält, wie das Bindungsverhalten durch Größe, Form, Oberflächenladung und umgebende Abwassermatrix (Chlorid, Sulfide, Tenside) beeinflusst wird und welche Parallelen zu anderen Nanopartikeln sichtbar sind (Limbach et al. 2008; Jarvie et al. 2009; El Badawy et al. 2010; Brar et al. 2010). Besonderes Interesse sollte der Speziierung von Silber im Abwasser gelten, denn die Löslichkeit der verschiedenen Silberformen bestimmt die Konzentration der freien Silberionen und damit über die unmittelbare Wirkung auf Organismen.

\section{Ziele}

Mit der vorliegenden Studie soll die Frage beantwortet werden, welchen Einfluss Nanosilber auf die Reinigungsleistung einer Kläranlage ausübt und wie es sich im System, insbesondere im Überschussschlamm und gereinigten Abwasser, verteilt. Der Verbleib und das Verhalten von partikulärem Silber werden in drei Teilprojekten untersucht:

1. Ermittlung der Nitrifikationsleistung von Belebtschlamm nach Zugabe von fünf verschiedenen Silberprodukten, davon vier Nanosilberarten (Hemmtests). Da Nitrifikanten besonders sensitiv auf Schadstoffe im Abwasser reagieren, wird der Einfluss von Silber quantifiziert.

2. Massenbilanz von Nanosilber in einer Pilotkläranlage (70 Einwohnerwerte). Die Untersuchung soll Auskunft geben, wie sich Nanosilber in der biologischen Reinigungsstufe verhält.

3. Vorkommen von Silber in einer realen kommunalen Kläranlage (60000 Einwohnerwerte).

Durch die Untersuchung wird erstmals eine vollständige Massenbilanz für Nanosilber experimentell erarbeitet und unter realen Anlagenbedingungen verifiziert.

\section{Material und Methoden}

\subsection{Hemmtests}

Zur Bestimmung der Nitrifikationsleistung im Belebtschlamm wurden Batchversuche durchgeführt. Silbernitrat wurde als Referenzsilber zu vier partikulären Silberformen (Tab. 1) bei Dosierungsmengen von $1 \mathrm{mg} / \mathrm{L}$ und $100 \mathrm{mg} / \mathrm{L}$ Ag getestet. Die vier Silberadditive sind Marktprodukte und werden in Textilien, Kunststoffen usw. eingesetzt. Für jede Variante wurden jeweils zwei Batchreaktoren (2-L-Glasbehälter) mit identischem, homogenisiertem Belebtschlamm bei konstant $+20^{\circ} \mathrm{C}$ betrieben. Der eine Reaktor stellt die Referenz zur untersuchten Silberart und -konzentration dar. Die Abbauleistung von Ammonium im verwendeten Schlamm der Kläranlage Kloten/Opfikon mit 4 g/L TS-Gehalt beträgt rund $10 \mathrm{mg} / \mathrm{L}$ Ammonium pro Stunde. Für partikuläres Silber wurde angenommen, dass hemmende Effekte erst nach längerer Expositionsdauer auftreten. Deshalb wurde die Wirkung von Silber auf den Ammoniumabbau (Zugabe von $20 \mathrm{mg} / \mathrm{L}$ Ammonium als Ammoniumstickstoff) sowohl nach $2 \mathrm{~h}$ als auch nach 6 Tagen Expositionsdauer (1 Schlammalter der Kläranlage Kloten/Opfikon) untersucht.

Der Schlamm in den Reaktoren wurde über die Versuchsdauer permanent gerührt (300 U/min), sodass sich durch die Belüftung eine für die Nitrifikation ausreichende Sauerstoffkonzentration $\left(4-6 \mathrm{mg} / \mathrm{L} \mathrm{O}_{2}\right)$ einstellte. Im Versuch mit 6 Tagen Expositionsdauer wurde der Schlamm nur gerührt. Damit lässt sich der Zerfall der Nitrifikanten vermindern (Siegrist et al. 1999). Zusätzlich wurde für $15 \mathrm{~min}$ pro Tag stoßweise belüftet, um eine anaerobe Vergärung des Schlamms zu verhindern.

Die Umsetzung des im Abwasser vorhandenen Ammoniums über Nitrit zu Nitrat wurde dadurch nachgewiesen, 
Tab. 1 Charakteristika von den getesteten vier Silberformen und Silbernitrat

\begin{tabular}{|c|c|c|c|c|c|}
\hline Testsubstanz & Form & $\begin{array}{l}\text { Größenbereich } \\
\text { (nm) }\end{array}$ & Beschichtung & Produkt & Hersteller \\
\hline Silbernitrat & Lösung & - & - & - & \\
\hline $\begin{array}{l}\text { Kolloidales } \\
\text { Nanosilber } A^{a}\end{array}$ & Suspension & $5-50$ & Emulgator $^{d}$ & AgPURE WS10 & $\begin{array}{l}\text { rent-a-scientist } \\
\text { GmbH }\end{array}$ \\
\hline $\begin{array}{l}\text { Kolloidales } \\
\text { Nanosilber B }\end{array}$ & Pulver & $5-50$ & Polymer & SmartSilver Pro & NanoHorizons, Inc. \\
\hline $\begin{array}{l}\text { Partikuläres } \\
\text { Silberchlorid }^{\mathrm{a}}\end{array}$ & Suspension & $20-500^{b}$ & Polymer & iSys AG & $\begin{array}{l}\text { CHT R. Beitlich } \\
\text { GmbH }\end{array}$ \\
\hline $\begin{array}{l}\text { Mikrokomposit- } \\
\text { Silber }\end{array}$ & Pulver & $5-50^{c}$ & - & HeiQ AGS-20 & HeiQ Materials AG \\
\hline
\end{tabular}

${ }^{a}$ Kolloidales Nanosilber und partikuläres Silberchlorid wurden in der Pilotkläranlage untersucht.

${ }^{\mathrm{b}}$ Die Silberpartikel weisen mit bimodaler Größenverteilung zwei Maxima bei 30 und $300 \mathrm{~nm}$ auf.

${ }^{c}$ Das Nanosilber liegt auf und in amorphen Silikatpartikeln vor, welche eine Größe von $>1000 \mathrm{~nm}$ aufweisen.

${ }^{d}$ Beschichtung mit nichtionogenem Polyoxyethylen-Fettsäureester. dass die Proben filtriert $(0,8-\mu \mathrm{m}$-Cellulosefilter) und mittels Fließinjektionsanalyse (Ammoniumstickstoff) und Ionenchromatografie (Nitrat, Nitrit) analysiert wurden. Über $2 \mathrm{~h}$ Versuchsdauer wurden alle 30 min Proben entnommen.

\subsection{Bilanzierung in Pilotkläranlage}

Die Bilanzierungsversuche wurden in einer Pilotkläranlage mit konstantem Abwasserzulauf von $24 \mathrm{~m}^{3} / \mathrm{Tag}$ durchgeführt (70 Einwohnerwerte), die aus zwei je $7 \mathrm{~m}^{3}$ großen unbelüfteten und belüfteten Belebungsbecken und einem Nachklärbecken mit $11 \mathrm{~m}^{3}$ Volumen besteht (Abb. 1). Täglich wird $1 \mathrm{~m}^{3} /$ Tag Überschussschlamm aus dem unbelüfteten Belebungsbecken abgezogen. Das durchschnittliche Schlammalter liegt bei 12 Tagen und der Gehalt an Trockensubstanz (TS) im Mittel bei $3 \mathrm{~g} / \mathrm{L}$ TS. In der Systembetrachtung wird die Vorklärung nicht berücksichtigt.

Das Verhalten von zwei Silberadditiven wurde untersucht: das kolloidale Nanosilber A mit enger Größenverteilung von 5-50 nm und das partikuläre Silberchlorid (Tab. 1). Die Silberchloridpartikel weisen eine bimodale Größenverteilung über eine Spannbreite von 20-500 nm auf. Die wei-

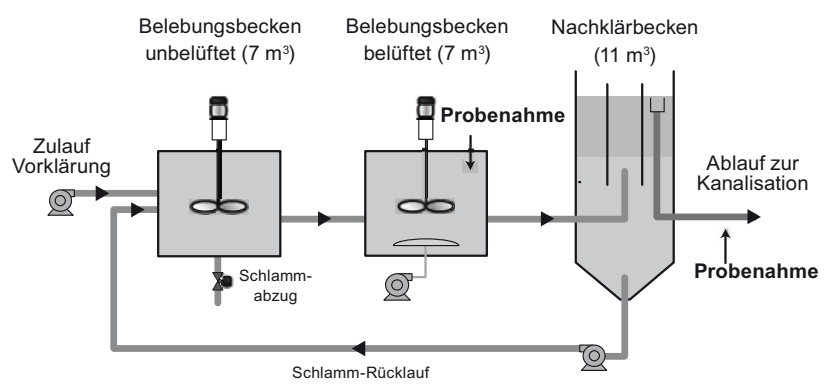

Abb. 1 Schema zur Pilotkläranlage mit zwei biologischen Reinigungsstufen und der Nachklärung. Die Dosierung erfolgte in das unbelüftete Belebungsbecken sowie die Probenahmen im belüfteten Becken und im Ablauf teren Additive (Stabilisatoren usw.) in beiden Suspensionen sind nicht bekannt. Die jeweiligen Suspensionen wurden wässrig verdünnt (deionisiertes Wasser) und in einem Vorlagebehälter konstant gerührt (120 L, Polypropylen, Rührwerk $200 \mathrm{rpm}$ ). Zur Einhaltung eines konstanten Druckniveaus während der Dosierung förderte eine Umwälzpumpe $(800 \mathrm{~L} / \mathrm{h})$ die verdünnte Suspension in einen Niveaubehälter. Kontinuierlich gravimetrisch erfasst und aufgezeichnet wurde der Gewichtsverlust des Vorlagebehälters.

Die Dosierung von Nanosilber erfolgte so, dass sich rasch ein quasi-stationäres Massengleichgewicht zwischen Zulauf-, Ablauf- und Schlammkonzentration einstellte. Dazu wurde in das unbelüftete Becken zunächst eine Silbermenge von $57,6 \mathrm{~g} / \mathrm{Tag} \mathrm{Ag}$ in Form von partikulärem Silberchlorid über $24 \mathrm{~h}$ dosiert (Abb. 1). Die Menge entspricht der anschließend vorgesehenen Zulauffracht über ein Schlammalter und ergab eine Zulaufkonzentration von $2400 \mu \mathrm{g} / \mathrm{L} \mathrm{Ag}$. Anschließend wurden während 24 Tagen (zwei Schlammalter) täglich $4,8 \mathrm{~g} \mathrm{Ag}$ über den Tag verteilt mit einer Schlauchpumpe automatisch dosiert. Unter Berücksichtigung der Abwassermenge im Zulauf resultiert eine Zulaufkonzentration von $200 \mu \mathrm{g} / \mathrm{L} \mathrm{Ag}$. Eine solche Zulaufkonzentration erlaubt eine elektronenmikroskopische Identifizierung des Nanosilbers im Belebtschlamm und im Ablaufwasser. Über die gesamte Versuchsdauer von 25 Tagen ergab sich eine dosierte Silbermenge von 172,8 g Ag. Im Zulauf wurde eine Hintergrundkonzentration von $1,5 \mu \mathrm{g} / \mathrm{L}$ Ag nachgewiesen. Diese Konzentration entspricht $<1 \%$ der Dosierungskonzentration und wird daher in der Bilanz vernachlässigt.

Die Beprobung des Überschussschlammes im belüfteten Belebungsbecken und des gereinigten Abwassers erfolgte in Abständen von 3-4 Tagen sowie während der Abklingphase alle 6 Tage (halbes Schlammalter). Mit der Probenahme in der Abklingphase wurde das Auswaschen des noch im System vorhandenen Silbers überwacht. Alle Proben lagerten in PET-Kunststoffflaschen $(200 \mathrm{~mL}$, mit $65 \%$ iger 
$\mathrm{HNO}_{3}$ angesäuert) bis zur Aufarbeitung im Kühlschrank (1-3 Tage).

Ein Teilstrom des gereinigten Abwassers wurde während der Abklingphase über eine Tuchfiltrationsanlage geleitet, mit dem Ziel, die verbleibende Fracht von den gesamten ungelösten Stoffen (GUS) und Silber zu reduzieren. In der Tuchfiltrationsanlage (Mecana Umwelttechnik AG, Reichenburg/CH) werden die Einzelfasern des Polstoffs schräg angeströmt und dadurch flachgedrückt, sodass eine mechanische Filtrationswirkung eintritt. Ablagerungen auf den Polfasern (Filterkuchen) erhöhen die Filterwirkung. Sinkt die Filtergeschwindigkeit unter einen festgelegten Wert, wird der Polfilter automatisch abgesaugt. Ungebundene Trübstoffe $<10 \mu \mathrm{m}$ Größe können den Filter „Polstoff Mikrofaser grün“ (Polyester, $750 \mathrm{~g} / \mathrm{m}^{2}$ ) passieren. Die Anlage wurde mit einem Zufluss von $330 \mathrm{~L} / \mathrm{h}$ betrieben. Über 5 Tage Versuchsdauer wurden täglich Tagesmischproben aus Zulauf, Ablauf und Spülwasser gesammelt.

Die chemische Analyse der Silberkonzentration erfolgte nach einem Mikrowellenaufschluss mit Königswasser im induktiv gekoppelten Plasma mit Massenspektrometrie (ICP-MS). In einem Ringversuch zur Silberanalytik von Abwasser- und Schlammproben wurde nachgewiesen, dass nur nach Königswasseraufschluss unter extremen Aufschlussbedingungen (Mikrowelle mindestens $10 \mathrm{~min}$ bei $+170^{\circ} \mathrm{C}$ ) Silberpartikel vollständig quantifiziert werden (nach $\mathrm{HNO}_{3}$ Aufschluss sind bis $50 \%$ weniger Ag nachweisbar).

Einzelproben wurden mit der Raster- oder Transmissionselektronenmikroskopie (REM, TEM) in Kombination mit energiedispersiver Röntgenfluoreszenzspektroskopie (EDX) untersucht. Damit lassen sich morphologische und chemische Informationen gewinnen. Für die Analysen wurden einerseits die Schlammproben abfiltriert (Polycarbonat, Nucleopore, 0,4 $\mu \mathrm{m}$ ) und die getrockneten Filter im REM untersucht, andererseits Ablaufproben direkt auf TEM-Träger zentrifugiert $(2 \mathrm{~h}, 3000 x \mathrm{~g})$ und ebenfalls elektronenmikroskopisch analysiert. Außerdem wurden TS-Gehalte mittels Filtration bestimmt. Dazu wurden jeweils die Feststoffe in $1 \mathrm{~L}$ Abwasser über Glasfaserfilter $(0,7 \mu \mathrm{m})$ filtriert und nach der Trocknung ausgewogen.

\subsection{Vorkommen in realer Kläranlage}

Die Kläranlage Kloten/Opfikon ist für eine Reinigungsleistung von 60000 Einwohnerwerten ausgelegt und ist nördlich von Zürich gelegen. Die Reinigungsanlage besteht aus Vorklärung, biologischer Reinigung im Belebtschlammverfahren mit Nitrifikation und Teildenitrifikation und einer nachgeschalteten Raumfiltration. Das Mischabwasser kommt aus Industrie, Gewerbe, Privathaushalten und vom Flughafen Zürich. Der tägliche Abwasserzufluss aus den zwei Einzugsgebieten Kloten und Opfikon beträgt bei Trockenwetter durchschnittlich $12000 \mathrm{~m}^{3} / \mathrm{Tag}$. Wie eigene Vorstudi- en ergaben, wird durch einen industriellen Indirekteinleiter über den Zufluss Opfikon nanoskaliges Silber mit nahezu konstanter Fracht zugeführt. Der Schlamm bleibt 17 Tage in der Faulung und täglich werden daraus durchschnittlich $4 \mathrm{t}$ TS ausgefaulter Schlamm abgezogen.

Proben wurden in vier Kampagnen jeweils von beiden Zuläufen, dem Ablauf nach der Sandfiltration und aus dem Faulschlamm entnommen. Bei den Wasserproben handelt es sich um abflussproportionale Tagesmischproben bei Trockenwetter. Der Faulschlamm weist eine durchschnittliche Aufenthaltszeit von 17 Tagen auf. Aus dem Faulschlamm sind die Proben zufällig entnommen worden. Wiederum wurden innerhalb weniger Tage die Proben $(200 \mathrm{~mL}$ Kunststoffflaschen, mit $65 \%$ iger $\mathrm{HNO}_{3}$ angesäuert) mit Mikrowelle aufgeschlossen (Königswasser) und mit ICP-MS analysiert.

\section{Ergebnisse und Diskussion}

\subsection{Einfluss von Nanosilber auf die Nitrifikationsleistung (Hemmtest)}

Der Abbau von Ammonium wurde immer im Vergleich zum Referenzreaktor ausgewertet. Der negative Verlauf der Trendlinie, in Abb. 2 für kolloidales Nanosilber A und Silbernitrat über $2 \mathrm{~h}$ Versuchsdauer dargestellt, verdeutlicht den zeitabhängigen Ammoniumabbau im Belebtschlamm (Abb. 2). Unterschiede zwischen Referenz- und Testreaktor von $<5 \%$ sind der Streuung bei solchen Versuchen zuzuordnen. Die vorgestellten Resultate veranschaulichen die hohe Konsistenz und Reproduzierbarkeit der standardisierten Methodik.

Auf dieser Basis wurde die relative Nitrifikationsleistung im Vergleich mit der Aktivität im Referenzschlamm für jede Silberart berechnet (Abb. 3). Die Zugabe von $1 \mathrm{mg} / \mathrm{L} \mathrm{Ag}$ in Form von Nanopartikeln, entsprechend $250 \mathrm{mg}$ Ag pro $\mathrm{kg}$ TS, ergab für alle vier Silberadditive keine messbare Hemmung des Ammoniumabbaus. Dies gilt sowohl für $2 \mathrm{~h}$ als auch für 6 Tage Expositionsdauer. Auch das zum Ver-
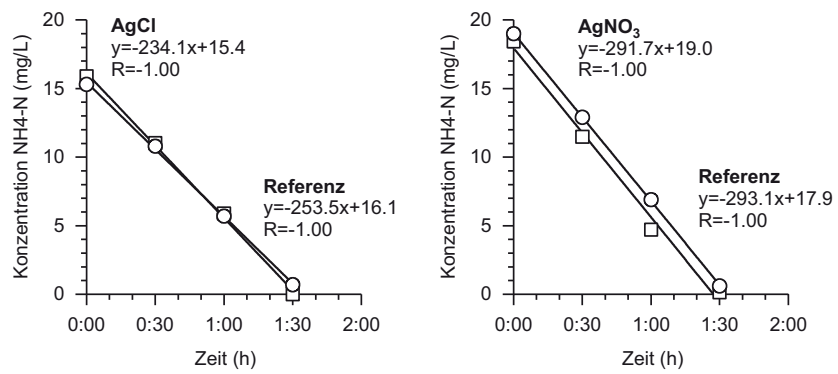

Abb. 2 Ammoniumabbau direkt nach Zugabe von $1 \mathrm{mg} / \mathrm{L} \mathrm{Ag}$ in Form von kolloidalem Nanosilber (links) und Silbernitrat (rechts) 
Abb. 3 Relative Nitrifikationsleistung des Belebtschlammes im Vergleich zum jeweiligen Referenzschlamm bei Zugabe von $1 \mathrm{mg} / \mathrm{L}$ und $100 \mathrm{mg} / \mathrm{L}$ Silber nach $2 \mathrm{~h}$ und 6 Tagen Expositionsdauer

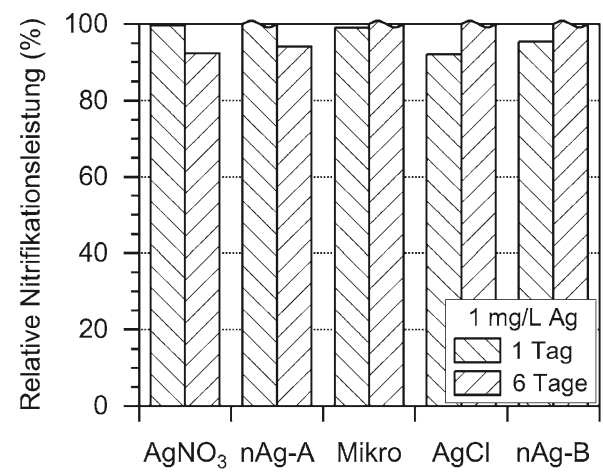

gleich verwendete Silbernitrat, welches ausschließlich aus Silberionen besteht, zeigte keine Beeinträchtigung (Abb. 3). Die geringen Abweichungen vom $100 \%$-Wert, z. B. für Mikrokomposit-Silber und partikulärem Silberchlorid nach 6 Tagen, lassen sich auf prozessbedingte Abweichungen im Reaktor zurückführen. Eine Dosierung von $1 \mathrm{mg} / \mathrm{L} \mathrm{Ag}$ in den Belebtschlamm entspricht bei einem Schlammalter von 12 Tagen und einer hydraulischen Aufenthaltszeit von 0,6 Tagen (= Pilotkläranlage) rund $50 \mu \mathrm{g} / \mathrm{L} \mathrm{Ag} \mathrm{im} \mathrm{Zulauf.} \mathrm{Firk}$ (1986) und Howe und Dobson (2002) konnten bis $50 \mathrm{mg} \mathrm{Ag} /$ kg TS keinen verminderten Ammoniumabbau nachweisen, wobei das Silber vermutlich nicht nanopartikulär vorlag.

Die Zugabe von $100 \mathrm{mg} / \mathrm{L} \mathrm{Ag}$ führte bei allen Silberformen zu einer eindeutigen Hemmung des Ammoniumabbaus (Abb. 3). Wiederum unter Berücksichtigung von 12 Tagen Schlammalter und 0,6 Tagen hydraulischer Aufenthaltszeit entspricht die Dosierung einer theoretischen Zulaufkonzentration von $5000 \mu \mathrm{g} / \mathrm{L} \mathrm{Ag}$. Die Nitrifikationsleistung wurde nach Zugabe von Silbernitrat zu $100 \%$ gehemmt, d.h. der Ammoniumabbau ist vollständig unterbunden und der von kolloidalem Nanosilber A zu 90\%. Mikrokomposit-Silber zeigte nach $2 \mathrm{~h}$ Exposition eine $60 \%$ ige Hemmung und nach 6 Tagen Expositionsdauer ebenfalls nahezu $100 \%$ Wirkung. Partikuläres Silberchlorid verband sich mit einem $50 \%$ igen Hemmeffekt ohne expositionsabhängige Wirkung. Die Zugabe des kolloidalen Nanosilbers B bedingte unter allen Silberformen mit 20-30\% die geringste Hemmung (Abb. 3). Möglicherweise kontrolliert hier die Polymerbeschichtung die Freisetzung von Silberionen am effizientesten.

Das Vorkommen von freien Silberionen, welche Einfluss auf die Nitrifikantentätigkeit haben könnten, wird zum einen durch die Chloridkonzentration im Belebtschlamm gesteuert. Unabhängig von der dosierten Silberkonzentration $(1$ oder $100 \mathrm{mg} / \mathrm{L} \mathrm{Ag}$ ) und der Art des Silberadditivs dürften bei einer Konzentration von $120 \mathrm{mg} / \mathrm{L}$ Chlorid nur rund $7 \mu \mathrm{g} / \mathrm{L}$ gelöstes Silber vorliegen. Zum anderen binden Liganden, welche als organische Komplexbildner, Huminsäuren oder reduzierte Schwefelgruppen im Abwasser auftreten, freie Silberionen (Howe und Dobson 2002; Luoma 2008; Scheckel et al. 2010). Aus Sulfidionen und
Silberionen entsteht nahezu unlösliches Silbersulfid. Wie nach Zugabe von $1 \mathrm{mg} / \mathrm{L} \mathrm{Ag} \mathrm{nachgewiesen,} \mathrm{werden} \mathrm{durch}$ diese Mechanismen produktspezifische Unterschiede unterbunden. Nanosilber bleibt also ohne messbaren Hemmeffekt auf Nitrifikanten, weil freie Silberionen stetig aus der Wasserphase entfernt werden. Der Befund nach Zugabe von $100 \mathrm{mg} / \mathrm{L} \mathrm{Ag}$ ist insofern überraschend. Angenommen wird, dass die potenziell verfügbaren Mechanismen bei einer derart hohen Silberdosierung zu langsam wirken und sich nicht das erwartete Lösungsgleichgewicht einstellt. Ob sich die Hemmung nur auf Silberionen oder auch auf Additive der Suspensionen zurückführen lässt, ist zudem unklar.

Die getestete Silberkonzentration von $100 \mathrm{mg} \mathrm{Ag} / \mathrm{L} \mathrm{Be}-$ lebtschlamm einer realen Kläranlage (4 g/L TS) entspricht einem Silbergehalt von $25000 \mathrm{mg} \mathrm{Ag} / \mathrm{kg}$ TS. In Klärschlämmen von Schweizer Kläranlagen wurden nur bis zu $100 \mathrm{mg} / \mathrm{kg} \mathrm{Ag}$ gefunden, wenn im Einzugsgebiet industrielle Indirekteinleiter angeschlossen waren (Bachmann et al. 1999). Die Zugabe von $1 \mathrm{mg} / \mathrm{L} \mathrm{Ag}$, welche $250 \mathrm{mg} \mathrm{Ag} / \mathrm{kg}$ TS entspricht, ist daher bereits als „worst case“ einzustufen und höhere Zugaben sind hinsichtlich ihrer Praxisbedeutung zu relativieren.

Choi et al. $(2008,2009)$ beobachteten bei Zugabe von $1 \mathrm{mg} / \mathrm{L} \mathrm{Ag}$ bereits eine signifikante Hemmung der Nitrifikationsleistung. Der dort eingesetzte aufgereinigte Belebtschlamm ohne potenzielle Liganden und Chlorid lässt aber auch erwarten, dass freie Silberionen vorliegen und diese zu einer Hemmung führen. Da sich nach Zugabe von einzelnen Liganden die Hemmwirkung reduzieren ließ (Choi et al. 2009), wird diese Annahme auch experimentell bestätigt. Die vorliegenden Resultate sind daher praxisnah, während die Studie von Choi et al. (2008) prozessorientiert angelegt und nicht auf reale Kläranlagen übertragbar ist.

\subsection{Pilotkläranlage}

Im Überschussschlamm nehmen die Konzentrationen über die Dosierungsphase von $3000 \mu \mathrm{g} / \mathrm{L}$ auf rund $5500 \mu \mathrm{g} / \mathrm{L}$ $\mathrm{Ag}$ zu und sinken über weitere zwei Schlammalter auf rund $1000 \mu \mathrm{g} / \mathrm{L} \mathrm{Ag}$ (Abb. 4). Die Anreicherung im Schlamm be- 


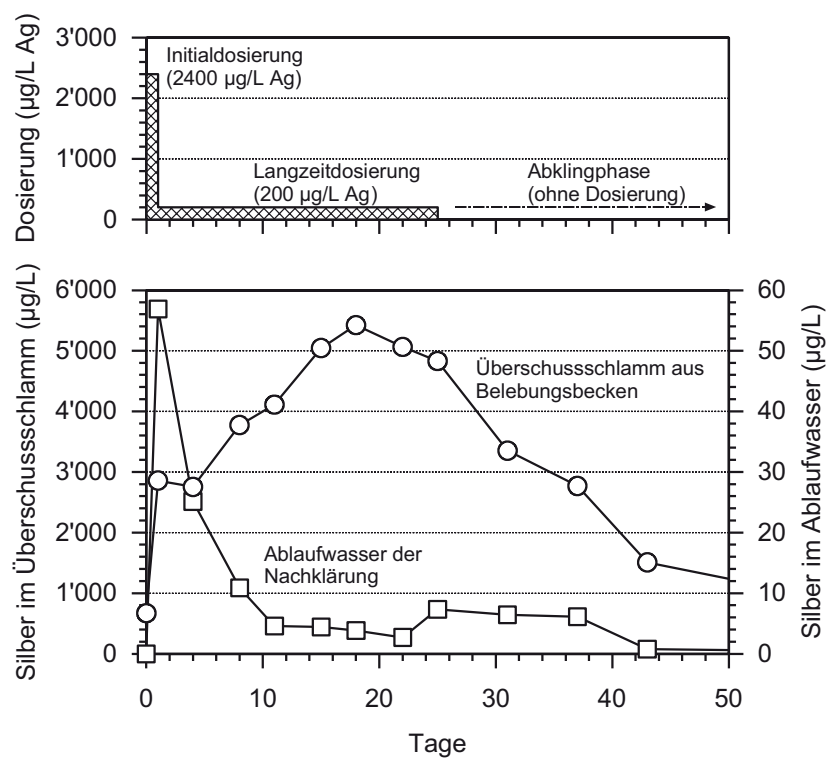

Abb. 4 Dosierung von partikulärem Silberchlorid (oben) sowie Silberkonzentrationen im Überschussschlamm (abgezogener Belebtschlamm) und im Ablaufwasser des Nachklärbeckens der Pilotkläranlage (unten). Die Initialdosierung erfolgte über 1 Tag und die Langzeitdosierung über 24 Tage mit jeweils $24 \mathrm{~m}^{3} / \mathrm{Tag}$ Abwasserzufluss

nötigt beinahe die gleiche Zeitspanne wie die Reinigung des Systems. Ganz anders verhält sich der Konzentrationsverlauf im Ablaufwasser des Nachklärbeckens. Unmittelbar nach der Initialdosierung tritt ein absolutes Maximum von rund $60 \mu \mathrm{g} / \mathrm{L} \mathrm{Ag}$ auf (24h nach Dosierungsbeginn). Anschließend gehen bei einer 24-tägigen konstanten Zulaufkonzentration von $200 \mu \mathrm{g} / \mathrm{L} \mathrm{Ag}$ die Ablaufkonzentrationen über 10 Tage auf $<5 \mu \mathrm{g} / \mathrm{L}$ zurück. Die Silberkonzentrationen bleiben bis Versuchsende (weitere rund 40 Tage) in diesem Bereich. Für diesen quasi-stationären Massenfluss resultiert für Zulauf- versus Ablaufkonzentration ein Faktor von 0,025 . Wird dieser Faktor auf eine in der Umwelt nachgewiesene mittlere Zulaufkonzentration von $4 \mu \mathrm{g} / \mathrm{L} \mathrm{Ag}$ bezogen (Shafer et al. 1998), so sind im Ablauf einer realen Kläranlage mit rund $0,1 \mu \mathrm{g} / \mathrm{L} \mathrm{Ag} \mathrm{zu}$ rechnen.

Basierend auf den gemessenen Konzentrationen von Silber und den erfassten Abwassermengen wurde der kumulative Austrag berechnet und auf die Dosiermenge $(172,8 \mathrm{~g} \mathrm{Ag})$ normiert (Abb. 5). Dabei wird deutlich, dass während der Dosierungsphase (erste 24 Tage) $60 \%$ Ag mit dem Überschussschlamm und $3 \%$ Ag über den Nachklärbeckenablauf das System verlassen. Über die folgende Ruhephase von zwei Schlammaltern (zweite 24 Tage), in der kein Silbereintrag erfolgte, wurden 35\% Ag mit dem Schlamm und 2\% Ag mit dem gereinigten Abwasser ausgetragen. Der Gesamtaustrag von $95 \%$ mit dem Schlamm und 5\% mit dem Ablaufwasser nach 49 Versuchstagen ergibt eine vollständige Wiederfindung. Identisch fällt nicht nur die Massenbilanz für das kollo-

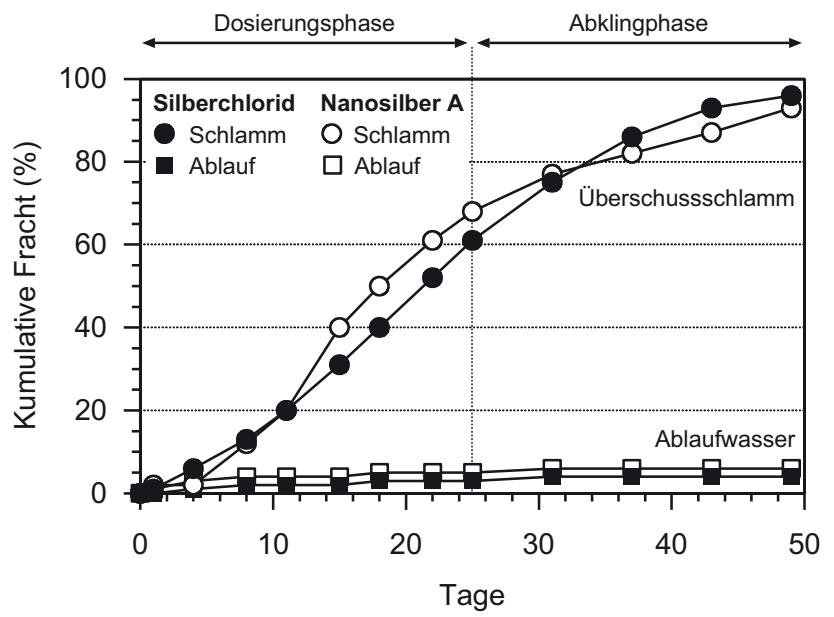

Abb. 5 Kumulativer relativer Austrag von Silber mit dem Überschussschlamm und Nachklärablauf aus der Pilotkläranlage nach Zugabe von partikulärem Silberchlorid und kolloidalem Nanosilber A

idale Nanosilber A aus, sondern vergleichbare Bilanzen werden auch von Shafer et al. (1998) für Gesamtsilber in Kläranlagen berichtet.

Während der Dosierungsphase stellt sich ein quasi-stationäres Massengleichgewicht über das System ein (nahezu linearer Verlauf der Summenkurven in Abb. 5). Die Silberkonzentrationen im Eintrag, Schlammaustrag und Ablauf erreichen einen konstanten Bereich, da alle Systembedingungen beibehalten werden. Unter dieser Vorrausetzung lässt sich eine durchschnittliche Tagesmassenbilanz für Silber und die Feststoffe erstellen. In die Pilotkläranlage gelangen 4,8 g/Tag Ag über den Zulauf. Davon wurden im täglich abgezogenen Schlamm ( $\left.1 \mathrm{~m}^{3} / \mathrm{Tag}\right)$ rund $4,7 \mathrm{~g} / \mathrm{Tag} \mathrm{Ag}$ analytisch nachgewiesen, die aus dem System abgezogen werden. Auf die im Schlamm enthaltenen $3 \mathrm{~g} / \mathrm{L}$ TS $(3000 \mathrm{~g} /$ Tag TS) bezogen resultieren Silbergehalte von $1,6 \mathrm{~g} \mathrm{Ag} / \mathrm{kg}$ TS. Im täglich abgeleiteten Abwasser $\left(23 \mathrm{~m}^{3} / \mathrm{Tag}\right)$ wurden weitere $0,1 \mathrm{~g} / \mathrm{Tag} \mathrm{Ag}$ gefunden. Diese Menge entspricht unter Berücksichtigung der TS-Fracht von $0,003 \mathrm{~g} / \mathrm{L} \mathrm{TS}$ (70 g/ Tag TS) einem Silbergehalt von rund $1,4 \mathrm{~g} \mathrm{Ag} / \mathrm{kg}$ TS. Aus der Gegenüberstellung von Silber- und Feststoffaustrag ist erkennbar, dass der Silberaustrag direkt proportional zum Feststoffaustrag erfolgt und daher im Überschussschlamm und im gereinigten Ablaufwasser bezogen auf TS sehr ähnliche Silbergehalte vorliegen.

REM-Untersuchungen bestätigen, dass nanoskalige Silberpartikel an den biologischen Flocken adsorbiert und inkorporiert vorliegen (Abb. 6). Diese Bindungsart wurde sowohl für Flocken im Überschussschlamm als auch im Ablaufwasser beobachtet. Der Nachweis der guten Bindung der Silberpartikel an den Belebtschlammflocken ist ebenfalls für das kolloidale Nanosilber A erbracht worden. Dagegen wurden nur vereinzelt Einzelpartikel nachgewiesen. 

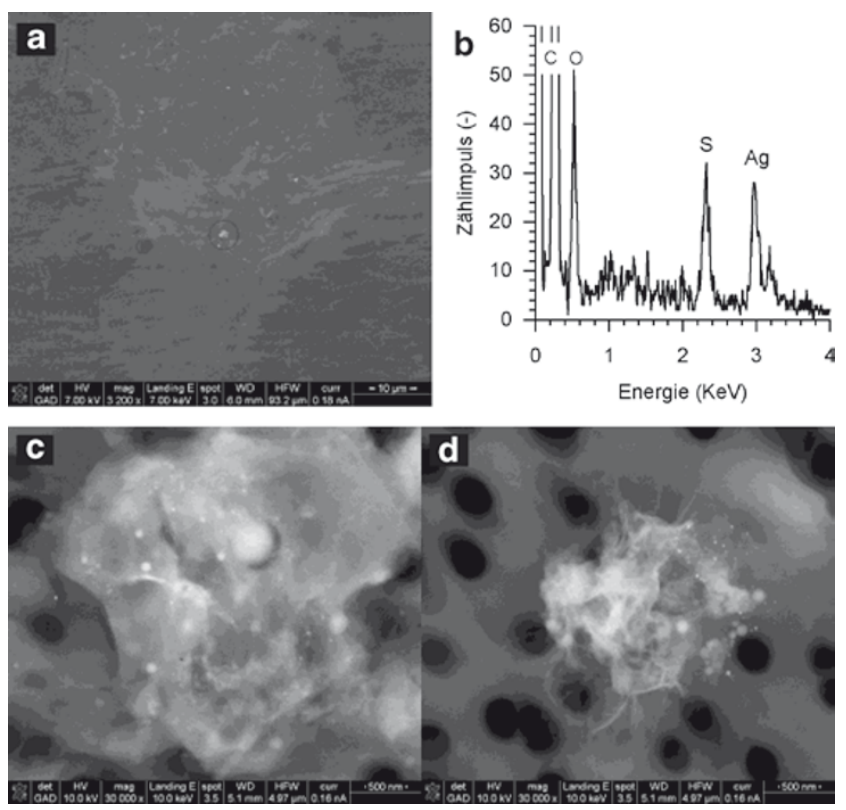

Abb. 6 REM-Bilder von partikulärem Silber nach Zugabe von Silberchlorid im Ablaufwasser (a) und dem EDX-Spektrum (b) sowie nach Zugabe von kolloidalem Nanosilber A gebunden an Schlammflocken vom Überschussschlamm (c) und gereinigtem Abwasser des Nachklärbeckens (d). Die weißen Punkte repräsentieren Silber, jeweils nachgewiesen mit der EDX

Einen Hinweis auf die tatsächlich vorkommende Bindungsform des Silbers im Ablaufwasser, welches zunächst als Silberchlorid dosiert wurde, liefert das EDX-Spektrum. Für analysierte Silberpartikel wurden Silber- und Schwefelsignale aufgenommen, aber das Chlorsignal fehlte. Das nanoskalige Silberchlorid scheint also vollständig mit schwefelhaltigen Gruppen gebunden und als schwerlösliche Silbersulfide vorzuliegen. Auch nach Zugabe von kolloidalem Nanosilber A, welches metallischem Silber $\left(\mathrm{Ag}^{0}\right)$ entspricht, kommt in EDX-Spektren Silber assoziiert mit Schwefel vor.

Zur weiteren Prüfung der nachgewiesenen Bindung von Nanosilber an den Feststoffen sowie zur Klärung, ob sich die Silberfracht durch eine GUS-Reduktion im Ablauf reduzieren lässt, wurde der Nachklärung nachgeschaltet eine Tuchfiltrationsanlage betrieben. Tatsächlich konnte gezeigt werden, dass sich nach Zugabe von partikulärem Silberchlorid (bimodale Größenverteilung zwischen $20-500 \mathrm{~nm}$, Tab. 1) mit der Tuchfiltration die Silberfracht im Ablauf der Pilotkläranlage, nach rund zwei Betriebstagen zur Konditionierung des Filters, um rund $30-60 \%$ reduzieren lie $\beta$ und damit vergleichbar gut wie die GUS-Fracht. (Abb. 7). Das kolloidale Nanosilber A mit einer Größe von 5-50 nm wurde dagegen vergleichbar gut, aber mit stabiler Rate von $40 \%$ eliminiert. Dieser Befund - Nanosilber ist vergleichbar gut eliminierbar wie größere Silberpartikel - erscheint zunächst überraschend, denn für Nanopartikel wird vielfach eine schlechtere Elimination als für größere Partikel erwartet. Tatsächlich ist aber die Anlagerung von Partikeln diffusionslimitiert, sodass kleine Silberpartikel besser als größere in Flocken wandern. Mit wenigen ungebundenen Silberartikeln verbinden sich im Vergleich mit vielen kleinen Silberpartikeln konzentrationsrelevante Silbermengen. Da die Porengröße des Tuchfilters im Mikrometerbereich liegt und freies Nanosilber den Filter immer passieren sollte, lässt sich die Reinigung in erster Linie auf die Bindung an Belebtschlammflocken zurückführen sowie allenfalls noch auf die gute Filterleistung des sich aufbauenden Filterkuchens auf dem Polstoff.

\subsection{Kläranlage Kloten/Opfikon}

In der Kläranlage Kloten/Opfikon wurden die beiden $\mathrm{Zu}-$ läufe, der Ablauf und der Faulschlamm viermal analysiert (Tab. 2). Die für den Industrieeinleiter abgeschätzte Silberkonzentration im Zulauf Opfikon liegt bei $10-20 \mu \mathrm{g} / \mathrm{L} \mathrm{Ag}$. Die Messwerte liegen tatsächlich in diesem Bereich und bestätigen somit, dass Silber regelmäßig in die Kläranlage gelangt. Durch Kenntnisse zur Abwasserqualität des Indirekteinleiters kann davon ausgegangen werden, dass nanopartikuläres Silber vorliegt. Die Zulaufkonzentrationen von
Abb. 7 Elimination (\%) von Silber und GUS durch die Tuchfiltration des Abwassers bezogen auf die Zulaufmenge. Dargestellt sind die Resultate aus dem Versuch mit partikulärem Silberchlorid (links) und kolloidalem Nanosilber A (rechts). *: Probe nicht genommen
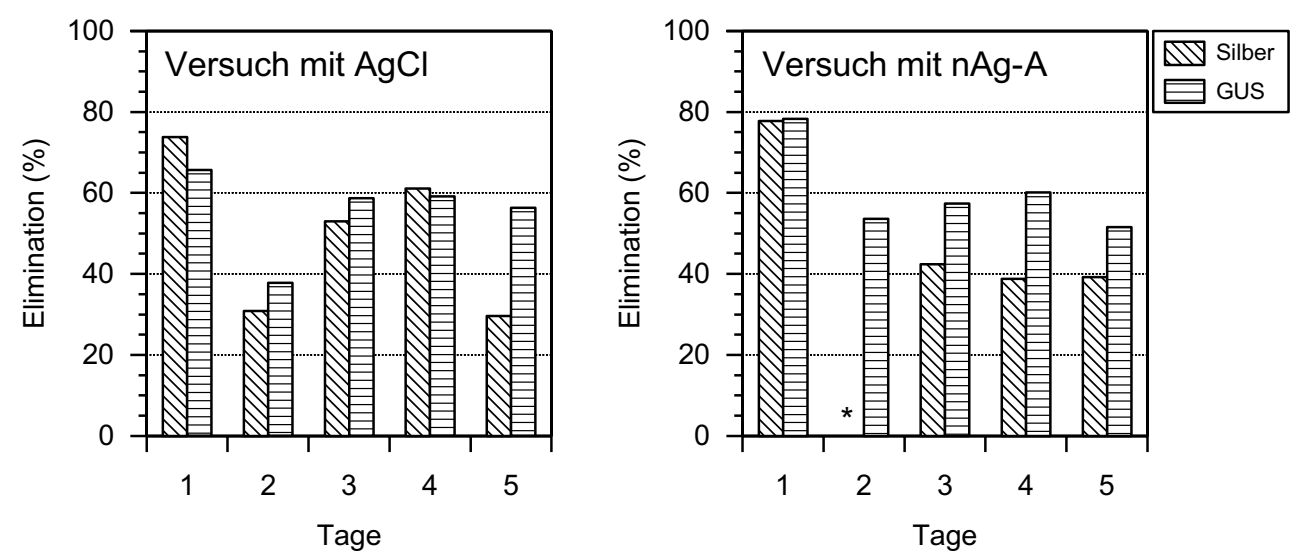
Tab. 2 Silberkonzentrationen und resultierende Frachten in der Kläranlage Kloten/Opfikon. Bei der Berechnung der Massenbilanzen wurde mit einer täglichen Schlammmasse von im Mittel $4 \mathrm{t} /$ Tag TS kalkuliert. Die geringen Abweichungen zwischen den Zulauffrachten und Ablauffrachten beruhen auf der Tatsache, dass zum einen der mittlere TS zugrunde gelegt wurde und zum anderen der Faulschlamm über 17 Tage integriert Silber akkumulierte, während die Abwasserproben nur eine Abflusskonzentration über $24 \mathrm{~h}$ widerspiegeln

\begin{tabular}{llcccc}
\hline Proben & Silber & \multicolumn{2}{c}{ Zulauf } & \multicolumn{2}{c}{ Austrag } \\
& & Opfikon & Kloten & Ablauf & Faulschlamm \\
\hline 1.1 & Konzentration $(\mu \mathrm{g} / \mathrm{L})$ & 14,0 & 1,9 & 0,54 & 870 \\
& Fracht $(\mathrm{g} / \mathrm{Tag})$ & 85 & 13 & $6(6 \%)$ & $116(94 \%)$ \\
1.2 & Konzentration $(\mu \mathrm{g} / \mathrm{L})$ & 18,4 & 1,6 & 0,19 & 860 \\
& Fracht $(\mathrm{g} / \mathrm{Tag})$ & 100 & 10 & $2(2 \%)$ & $115(98 \%)$ \\
$2.1^{\mathrm{a}}$ & Konzentration $(\mu \mathrm{g} / \mathrm{L})$ & 12,3 & 5,3 & 0,08 & 740 \\
& Fracht $(\mathrm{g} / \mathrm{Tag})$ & 62 & 31 & $0,9(1 \%)$ & $96(99 \%)$ \\
$2.2^{\mathrm{a}}$ & Konzentration $(\mu \mathrm{g} / \mathrm{L})$ & 12,3 & 2,5 & 0,07 & 580 \\
& Fracht $(\mathrm{g} / \mathrm{Tag})$ & 64 & 15 & $0,7(1 \%)$ & $76(99 \%)$ \\
& & & &
\end{tabular}

a Ab diesem Zeitpunkt wurde vom Einleiter die Anwendung von Silberchlorid reduziert, sodass Proben 2.1 und 2.2 die neue Situation widerspiegeln.
Kloten sowie die Hintergrundbelastung im Zulauf der Pilotkläranlage veranschaulichen, dass durch diffuse Einleitungen ins Abwasser (keine Indirekteinleiter) demgegenüber mit einer um rund 5-10-mal geringeren Silberkonzentration zu rechnen ist.

Im gereinigten Abwasser dagegen liegen die Konzentrationen teils nur geringfügig höher als die Bestimmungsgrenze $(0,05 \mu \mathrm{g} / \mathrm{L} \mathrm{Ag})$ mit einem Maximalwert von $0,5 \mu \mathrm{g} / \mathrm{L} \mathrm{Ag}$. Auch diese Silberkonzentrationen entsprechen Gesamtgehalten nach einem Totalaufschluss und sind nicht mit dem ökotoxikologisch relevanten ionischen Silber gleichzusetzen. Im frischen Faulschlamm wurden Konzentrationen von $500-900 \mu \mathrm{g} / \mathrm{L} \mathrm{Ag}$ bestimmt, die auf TS bezogen rund 20-30 mg Ag/kg TS entsprechen.

Die Silberfracht stellt die wesentliche Grundlage für die Massenbilanz sowie die Eliminationsrate dar. Zu erkennen ist eine Abnahme der Silberfracht im Zulauf Opfikon für die Proben 2.1 und 2.2 bezogen auf die vorherigen beiden Messkampagnen. Die Reduktion erklärt sich aus einer verringerten Silberanwendung beim Indirekteinleiter.

Vom gesamten Silbereintrag gelangen $94-99 \%$ in den Schlamm - und damit in der Schweiz in die Verbrennung - und 1-6\% Ag über die Nachklärung in den Vorfluter. Die Stoffströme fallen identisch zur Massenbilanz des partikulären Silberchlorids und kolloidalen Nanosilbers A in der Pilotkläranlage aus. Die Resultate der Pilotanlage werden also durch die Untersuchungen auf einer realen Kläranlage, in die überwiegend Silber in Form nanoskaligen Silbersulfids eingetragen wird, bestätigt und unterstreichen, dass Nanosilber in Kläranlagen durch Adsorption und Inkorporation in die Belebtschlammflocken effizient eliminiert wird. Die aus den Betriebsdaten der Kläranlage resultierende durchschnittliche Silberfracht in den Vorfluter beträgt jährlich $0,25-2,5 \mathrm{~kg} / \mathrm{a}$ $\mathrm{Ag}$. Auf das 60000 Einwohneräquivalent der Kläranlage Kloten/Opfikon bezogen erreichen jährlich $4-40 \mathrm{mg} / \mathrm{a} \mathrm{Ag}$ pro Einwohnerwert das Gewässer. Diese Abschätzung liegt 20-200-mal niedriger als das Resultat von Firk (1986).
Die Kläranlage verfügt über eine Sandfiltration nach der Nachklärung, wodurch eine zusätzliche Reduzierung der sonst zu erwartenden TS-Ablauffracht erreicht wird. Diese Maßnahme ist geeignet, wie die Versuche auf der Pilotkläranlage zeigen, auch gebundenes Nanosilber aus dem gereinigten Abwasser zu entfernen. Im Fall der Kläranlage Kloten/Opfikon wird erwartet, dass durch den Sandfilter rund zwei Drittel des Silbers aus dem Ablauf der Biologie zusätzlich entfernt werden. Eine parallel zu Silber erstellte Titanbilanz lässt die Annahme zu, dass die größeren Titandioxidpartikel (vermutlich in erster Linie Weißpigmente mit 300-400 nm Größe) die Kläranlage besser passieren als Nanosilber. Einer Zulaufkonzentration von $240 \mu \mathrm{g} / \mathrm{L}$ Ti stand eine Ablaufkonzentration von $200 \mu \mathrm{g} / \mathrm{L}$ Ti gegenüber $(20 \%$ Elimination). Die Konzentrationen im Faulschlamm lassen für Titan (in Form von Titandioxid) eine 10-mal geringere Elimination als für Silber (in Form von Silberpartikeln) erwarten.

Die Resultate zur Bindungsform des Silbers im Zufluss von Opfikon und dem Faulschlamm deuten wie schon in der Pilotkläranlage (Schlamm, Ablaufwasser) klar darauf hin, dass Nanosilber vollständig mit Schwefel koordiniert vorliegt (organisch oder anorganisch). Sollte im Ablaufwasser Silber tatsächlich vollständig als Silbersulfid vorliegen, kann ausgeschlossen werden, dass problematische Silberionen im Einleitegewässer freigesetzt werden.

\section{Schlussfolgerungen}

Unter Berücksichtigung, dass die jeweils untersuchte (dosierte) Silberform in realen Abwässern verschiedenen Umwandlungsprozessen unterliegt, lässt sich das Verhalten von Nanosilber in Kläranlagen wie folgt charakterisieren:

- Nanosilber ist im Abwasser nicht frei vorliegend, sondern wird in der biologischen Reinigungsstufe an Belebtschlammflocken gebunden. Nanopartikuläres Silber, 
wie es in der Studie untersucht wurde, wird demnach im biologischen Abwasserreinigungsprozess ausgezeichnet in die Schlammflocken an- und eingelagert. Liegen aber Silberpartikel vor, die grösser als Nanosilber im engeren Sinne sind (>100 nm), so ist möglicherweise das Bindungsvermögen herabgesetzt und die Eliminationseffizienz sinkt.

- Die Elimination in Kläranlagen beträgt $>95 \%$. Die Eliminationsrate von nanopartikulärem Silber bewegt sich im gleichen Bereich wie bei Untersuchungen vom Gesamtsilber. Im Vergleich mit anderen problematischen organischen Mikroverunreinigungen (Pharmaka, Pestizide, Biozide, andere Schwermetalle) ist die Elimination von Nanosilber als hoch einzustufen. Der pharmazeutische Wirkstoff Diclofenac wird beispielsweise nur zu 25\% eliminiert; Kupfer und Zink zwischen 60 und $80 \%$; sogar ohne jede Elimination passiert Carbamazepin die Kläranlage (Gälli et al. 2009). In der Schweiz sollen Kläranlagen so weit ausgebaut werden, dass mindestens eine $80 \%$ ige Elimination für Mikroverunreinigungen erreicht wird - für Nanosilber wird diese Anforderung bereits deutlich übertroffen.

- Eine Hemmung der Nitrifikationsleistung ist auch unter realitätsnahen Worst-Case-Bedingungen $(1 \mathrm{mg} / \mathrm{L} \mathrm{Ag}$ im Abwasser bzw. $250 \mathrm{mg} \mathrm{Ag} / \mathrm{kg}$ TS im Klärschlamm) nicht zu erwarten, da praktisch keine freien Silberionen vorliegen. Die vorliegenden Resultate und Angaben aus der Literatur unterstreichen, dass das ökotoxisch relevante ionische Silber in der realen Abwassermatrix zu unlöslichem Silberchlorid und Silbersulfid umgewandelt wird.

- Auch Nanosilber wandelt sich, wie erste Analysen mittels EDX andeuten, rasch und vollständig in unlösliches Silbersulfid um. Erste orientierende Resultate zeigen, dass bereits im Zulauf zur Kläranlage Silber mit Schwefel koordiniert vorliegt. Silbersulfid ist ökotoxisch anders einzuschätzen als gut lösliche Silberverbindungen.

- Die Nanosilbermenge, die über das Abwasser die Kläranlage verlässt, korreliert stark mit dem TS-Gehalt. Wird die GUS-Fracht im Abwasser reduziert, und darauf weisen Versuche mit einer Tuchfiltrationsanlage hin, lässt sich die Restsilberfracht im Ablauf der Kläranlage zusätzlich effizient reduzieren.

- In der Schweiz wird der entwässerte Klärschlamm in der Verbrennung entsorgt. Die angereicherte Silbermenge gelangt nicht in die Umwelt zurück. In Ländern, in denen Klärschlamm noch in der Landwirtschaft ausgebracht wird, führt das Silbersulfid zu einer Erhöhung der Silbergehalte im Boden. Aus heutiger Sicht sind aber die Silberfrachten als gering einzustufen und gegenüber geogenen Hintergrundwerten eher von untergeordneter Bedeutung.

\section{Empfehlungen und Ausblick}

Wie die Resultate klar zeigen, verbindet sich das Vorkommen von Nanosilber im Abwasser mit keinem akuten Handlungsbedarf. Das Risiko für eine Gewässerbelastung und Schädigung von aquatischen Organismen kann als gering eingestuft werden. Dagegen sollten dem Vorsorgeprinzip folgend Maßnahmen zur Emissionsminderung immer geprüft werden.

Da zahlreiche organische Mikroverunreinigungen, insbesondere polare, in Kläranlagen deutlich schlechter als Nanosilber eliminiert werden, sollten Maßnahmen zur Behandlungsoptimierung bevorzugt in diese Richtung getrieben werden. Wird im Rahmen eines Ausbaus von Kläranlagen aber eine weitergehende Reduktion der gesamten ungelösten Stoffe im Ablaufwasser angestrebt, so ließen sich infolgedessen auch Nanosilbereinträge in Gewässer weiter minimieren. Als technische Maßnahmen bieten sich dafür, wie bei der Kläranlage Kloten/Opfikon, neben Raumfiltern (Sandfilter) auch Flächenfilter, beispielsweise in Form von Trommelfiltern, an. Die heute zu beobachtenden niedrigen Ablaufwerte von Silber und das Vorkommen von fast unlöslichen Silberverbindungen stellen aber solche zusätzlichen Investitionskosten infrage, wenn nur die Kontamination durch Nanosilber im Vordergrund stehen würde. Falls in Zukunft nichtflockulierbare oberflächenstabilisierte Nanopartikel in Produkten eingesetzt und ausgewaschen werden, sollte der Handlungsbedarf neu beurteilt werden.

Publizierte Literaturdaten aus Laborversuchen sind meistens nicht geeignet, das Verhalten von Nanosilber in halbund großtechnischen Kläranlagen vorherzusagen. Diesbezüglich muss insbesondere der komplexen Abwassermatrix in Kläranlagen Beachtung geschenkt werden, da andernfalls versuchsbedingte Artefakte entstehen. Dabei ist immer zu berücksichtigen, dass in der Regel im Laborversuch die hydraulischen Aufenthaltszeiten und die Bildung von Belebtschlammflocken nicht der Realität entsprechend abgebildet werden können. Resultate aus Laborversuchen sind aber geeignet, beispielsweise den Einfluss bestimmter Liganden auf das Bindungsvermögen von Nanosilber prozessbezogen zu beschreiben.

Angeregt wird, die Forschung einerseits stärker auf Nanomaterialien zu lenken, die in Kläranlagen weniger gut eliminierbar sind als Nanosilber, und andererseits auf das Verhalten von Nanomaterialien in den festen Abfällen. In solchen Studien sind die Partikelgröße, die -morphologie sowie die Funktionalisierung der Partikeloberfläche stärker zu berücksichtigen.

Danksagung Die Elimination von nAg-A mit der Tuchfiltration wurde von unserem Kollegen Markus Behl erarbeitet - vielen Dank dafür. Für die ausgezeichnete Zusammenarbeit mit den Firmen CHT R. Beitlich GmbH, Tübingen (Deutschland) und HeiQ Materials AG, Bad Zurzach (Schweiz) möchten wir uns herzlich bedanken. Ganz beson- 
ders danken möchten wir der Projektbegleitgruppe, bestehend aus Bettina Hitzfeld und Christian Pillonel vom BAFU sowie Jesper Hansen und Peter Spohn vom AWEL. Das Projekt wurde durch das Schweizer Bundesamt für Umwelt (BAFU), Bern, und das Amt für Abfall, Wasser, Energie und Luft (AWEL), Zürich, gefördert.

\section{Literatur}

AWEL (2010) Persönliche Mitteilung durch Dr. Peter Spohn, Amt für Abfall, Wasser, Energie und Luft, Sektion Abwasserreinigungsanlagen. Zürich

Bachmann HJ, Berset JD, Candinas T, Chassot GM, Herren D, Kupper T (1999) Beobachtung des Stoffwechsels der Anthroposphäre im Einzugsgebiet ausgewählter Abwasserreinigungsanlagen (SEA) - Ergebnisse des Projekts SEA. Eid. Forschungsanstalt für Agrarökologie und Landbau, Institut für Umweltschutz und Landwirtschaft, Liebefeld, Bern

Benn T, Westerhoff P (2008) Nanoparticle silver released into water from commercially available sock fabrics. Environ Sci Technol 42:4133-4139

Blaser SA, Scheringer M, MacLeod M, Hungerbühler K (2008) Estimation of cumulative aquatic exposure and risk due to silver: contribution of nano-functionalized plastics and textiles. Sci Total Environ 390:396-409

Brar SK, Verma M, Tyagi RD, Surampalli RY (2010) Engineered nanoparticles in wastewater and wastewater sludge - evidence and impacts. Waste Manag 30:504-520

Burkhardt M, Zuleeg S, Etter B, Marti T, Landert L, Eugster J, Boller M, Kägi R, Sinnet B, Siegrist H, Vonbank R, Brunner S, Simmler H, Wichser A, Ulrich A, Seiler C, Haag R, Schmid P, Gerecke A, Vonmont H (2009) Nanopartikel in Fassadenbeschichtungen Charakterisierung, Auswaschung und Vergleich mit organischen Bioziden. Eawag, Dübendorf

Choi O, Deng KK, Kim N-J, Ross L, Surampalli RY, Hu Z (2008) The inhibitory effects of silver nanoparticles, silver ions, and silver chloride colloids on microbial growth. Water Res 42(12):30663074

Choi O, Clevenger TE, Deng B, Surampalli RY, Ross L, Hu Z (2009) Role of sulfide and ligand strength in controlling nanosilver toxicity. Water Res 43(7):1879-1886

El Badawy AM, Luxton TP, Silva RG, Scheckel KG, Suidan MT, Tolaymat TM (2010) Impact of environmental conditions ( $\mathrm{pH}$, ionic strength, and electrolyte type) on the surface charge and aggregation of silver nanoparticles suspensions. Environ Sci Technol 44:1260-1266

Europäische Union (1998) Richtlinie 98/8/EG des europäischen Parlaments und des Rates vom 16. Februar 1998 über das Inverkehrbringen von Biozid-Produkten. http://eur-lex.europa.eu/LexUriServ/ site/de/oj/1998/1 123/1 12319980424de00010063.pdf

Firk W (1986) Schwermetalle in Abwasser und anfallenden Schlämmen - Bilanzierung auf drei Kläranlagen. Schriftenreihe Gewässerschutz Wasser Abwasser 85, Gesellschaft zur Förderung der Siedlungswasserwirtschaft an der RWTH Aachen e.V. Aachen, S 467-483

Gälli R, Ort C, Schärer M (2009) Mikroverunreinigungen in den Gewässern - Bewertung und Reduktion der Schadstoffbelastung aus der Siedlungsentwässerung. Umwelt-Wissen 0917, Bundesamt für Umwelt, Bern
Geranio L, Heuberger M, Nowack B (2009) The behavior of silver nanotextiles during washing. Environ Sci Technol 43:8113-8118

Gorensek M, Recelj P (2007) Nanosilver functionalized cotton fabric. Textile Res J 77:138-141

Howe PD, Dobson S (2002) Silver and silver compounds: environmental aspects. Concise International Chemical Assessment Document 44, World Health Organization, Geneva

Hund-Rinke K, Simon M, Marscheider-Weidemann F, Kemper M (2008) Beurteilung der Gesamtumweltexposition von Silberionen aus Biozid-Produkten. Forschungsbericht 36004 020, Umweltbundesamt, Dessau-Roßlau

Jarvie HP, Al-Obaidi H, King SM, Bowes MJ, Lawrence MJ, Drake AF, Green MA, Dobson PJ (2009) Fate of silica nanoparticles in simulated primary wastewater treatment. Environ Sci Technol 43:8622-8628

Kägi R, Sinnet B, Zuleeg S, Hagendorfer H, Müller E, Vonbank R, Boller M, Burkhardt M (2010) Release of silver nanoparticles from outdoor facades. Environ Pollut 158:2900-2905

Kumar R, Howdle S, Münstedt H (2005) Polyamide/silver antimicrobials: effect of filler types on the silver ion release. J Biomedical Material Res Part B: Appl Biomaterials (75B):311-319

Lee HJ, Yeo SY, Jeong SH (2003) Antibacterial effect of nanosized silver colloidal solution on textile fabrics. J Material Sci 38:21992204

Limbach LK, Bereiter R, Müller E, Krebs R, Gälli R, Stark W (2008) Removal of oxide nanoparticles in a model wastewater treatment plant: influence of agglomeration and surfactants on clearing efficiency. Environ Sci Technol 42:4133-4139

Luoma SN (2008) Silver Nanotechnologies and the Environment Old Problems or New Challenges. PEN 15, Wilson Center and The Pew Charitable Trusts, Washington DC

Morones JR, Elechiguerra JL, Camacho A, Holt K, Kouri JB, Ramírez JT, Yacaman MJ (2009) The bactericidal effect of silver nanoparticles. Nanotechnology 16:2346-2353

Müller NC, Nowack B (2008) Exposure modeling of engineered nanoparticles in the environment. Environ Sci Technol 42:4447-4453

Ratte HT (1999) Bioaccumulation and toxicity of silver compounds: a review. Environ Toxicol Chem 18:89-108

Scheckel KG, Luxton TP, El Badawy AM, Impellitteri CA, Tolaymat TM (2010) Synchrotron speciation of silver and zinc oxide nanoparticles aged in a kaolin suspension. Environ Sci Technol 44:1307-1312

Shafer MM, Overdier JT, Armstrong DE (1998) Removal, partitioning, and fate of silver and other metals in wastewater treatment plants and effluent-receiving streams. Environ Toxicol Chem 17:630-641

Siegrist H, Brunner I, Koch G, Phan LC, Le VC (1999) Reduction of biomass decay rate under anoxic and anaerobic conditions. Water Sci Technol 39(1):129-137

Silverinstitute (2010) www.silverinstitute.org/supply demand.php

Som C, Halbeisen M, Köhler A (2009) Integration von Nanopartikeln in Textilien - Abschätzungen zur Stabilität entlang des textilen Lebenszyklus. Empa-Bericht, St. Gallen

Warila J, Batterman S, Passino-Reader DR (2001) A probabilistic model for silver bioaccumulation in aquatic systems and assessment of human health risks. Environ Toxicol Chem 20:432-441

Wen L-S, Santschi PH, Gill GA, Paternostro CL, Lehman RD (1997) Colloidal and particulate silver in river and estuarine waters of Texas. Environ Sci Technol 31:723-731 